\title{
Analyse einer neu entdeckten Mineralquelle zu Dürkheim an der Haardt;
}

im Auftrage der Königlich Bayer'schen Regierung des Rheinkreises ausgeführt

ron

Johann Eduard Herberger.

\section{Skizzirte Beschreibung der Gegend nebst geschicht- licken Bemerkungen.}

In der anmulhigsten Gegend der Rheinpfalz, am Eingange eines romantisch-schönen, durch den Griffel der Geschichte für alla Zukunft bewahrten, Thales liegt die Stadt Dürkheim *), angelelint an die Vorhiigel der Haardit.

Dem grossen Panorama dieser Landschaft mangelt der Glanz der Silberwogen eines majestälischen Stromes, - denn fernbin nur durchfurcht der weinumrankte Rhein die Gauen, - aber ler grüne Schmelz herrlicher Wiesen, und der gold'ne Teppich ïppiger Fruchtsaaten in östlicher Fiiclıtung, beut das Bild einer grossen Seeflüche, und die gartenähnlichen, ringsumber ausgebreitcten, Wein-und Obstanlagen, - ein Himnel, der selbst der südlichen Mandel Gedeihen verleiht. - Wer gelïstete nicht, die Geniisse, die diesc lachende Schöpfung darbietet, zu kosten?

*) Ehedem: Thüringeheim, Durinheim, Durinkeim, Durinkheim, Turenkheim, Dorenkheim u. s. f. Vergl. hierüber: „Joh. Georg Lehmann's geschichtliche Gemülde aus dem Rheinkreise Bayern's. Zweites Heft: Das Dïrkheimer Thal. Heidelberg, in Commission von G. Reichard; 183t. 80"6 welches Werk als Grundlage obiger Skizze gedient hat.

H. 
Hüher ragen gen Westen erst Hügel, dann die Berge der Haardt. Von den erslern lerab blickt die schlanke Rebe, und auf den Wipfeln breiten Kastanienwälder ihre schattenreichen Kronen aus; im Hintergrunde kleidet das nüchtliche Gewand von Nadelbölzern die Bergesrïcken, und mächtige Felsenpfeiler streben empor, und begrüssen den Wanderer mit ihrem kahlen, nacklen Haupte.

Gleichfalls in westlicher Richtung zieht sich von der Gemarkung der Sladt an zwischen Gebirgswänden das s. g. Durliheimer Thal. Die Zinnen der ehrwürdigen Abtey Limburg bewachen noch heute den Eingang dieses bilderreichen Thales, auf das wir zurückkommen werden.

Dürkheim zählt gegenwärlig 500 Häuser und ohngefähr 4500 Bewohner von munter-geschüftigem Charakter und Streben.

Wir wissen nicht, wer diesem Orle das Daseyn geschenkt hat. Der Leser belächelt vielleicht die Sage uns'rer Ahnen, die uns versichert, ein bei der Zerstörung llion's entronnener Fürst, Grunius, sey der Stifter Dürkheim's gewesen! Die begründetere Geschichte desselben reicht nur bis in's zelnne Jahrhundert, zu welcher Zeit es im Besitze der rheinfränkischen Herzoge gewesen zu seyn scheint. Später gebot ihm der Hirtenstab des Ables von Limburg. Ihm strillen die Sölne des Dürkheimer Thales, die Grafen von Leiningen, in blutigen Fehden die Rechte ab; auch die Einwohner suchten sich allmählig der auf ihnen lastenden Leibeigenschaft zu entfesseln, und dieses Gewirre von Dramen gebar einen rastlosen Wechsel von Kämpfen, gebar selbst mehrmalige Verwüstung und Einäscherung der Stadt. Auch der bluttriefende $A$ ar der französischen Légionen breitete seine Fitlige über diese, vom götllichen Hauche berïhrten, Gauen aus. Doch der Verwirrung entstieg endlich klar und unvergänglich die bïrgerliche Selbstständigkeit, 
und ein erfreulicher Wohlstand ist nunmehr die scliönste Lobrede auf den Schutz der Krone Bayerns.

Dürkheim besitzt zur Zeit ein Krankenhaus nebst einem kleinen Pfründner-Spitale, eine lateinische Schule, zwei Privat-Erziehungs-Institute; es ist der Sitz melurer königl. und städtischer Behörden, und in kurzer Entfernung - ge'n Osten - liegt die einzige Saline Rheinbayerns, Philippshalle, über welche ich zu eirter andern Zeit ausführlicher zu berichten gedenke.

Eine Anstalt ferner, die wir hier näber in's Auge fassen wollen, ist die Bade-Anstalt des Herrn Oberle. Dieser Privatmann grïndete im J. $183_{2}$ ein russisches Dampfbad, verbunden mit Sool-, Schwelel - und gewöhnlichen Bädern. Unfern der Saline nämlich quillt ein Wasser hervor, welches etwas Schwefelwasserstoffgas aushaucht, und es kann bei der vorzüglichen Lage Dürklieims, auch abgesehen von den Bestandtheilen des Wassers, nicht befremden, wenn wir dasselbe rom Geiste der Spekulation benutzt finden. Jedenfalls verdient das erwähnte Unternelımen nicht nur die Beachtung, sondern auch den Dank des Publikums. Dieses Wasser aber ist es, welches den Gegenstand dieser Abhandlung der Hauptsache nach ausmacht, und worüber ich mich in den nachfolgenden Abschnitten verbreiten werde *).

Doch ehe ich dazu übergehe, mag es vergönnt seyn, die begonnene Wanderung durch die Umgegend Dürkheims fortzusetzen und zu beendigen. Denn, wenn es wahr ist, dass die Heilkraft der Bäder nicht nur in ihrem chemischen Bestande, sondern auch in den physischen Polenzen beruht, welche aus den Vorziigen der Lage, der Umgebung, der

*) Die ganze Anstalt steht, wie ich vernommen habe, unter ärztlicher Aufsicht. 
Vergnügungen und Zerstreuungen hervorgehen: so erheischt jede Badbeschreibung auch eine, wenigstens kurze, Skizze derselben, um den Leser auf den Standpunkt klarer und vorurtheilsireier Würdigung zu erheben.

Wir haben bereits die Nachbarschaft der Saline, einer, durch ihre natürlichen und künstlichen Verhältnisse gleich interessanterı, als nützlichen Anstalt berührt.

Nürdlich von der Saline liegt das freundliche Pfaffingen, einst der Sitz der Grafen von Holucnburg, in dessen Gemarkung auch die nahe gelegenen, durch die Vortrefflichkeit ihrer Wein - Erzeugnisse ausgezeichneten, Dorfschaften: Ungstein, Kallstadt, und das gegen Süden gelegene $E l$ lerstadt gehörten.

Westlich von diesem freundlichen Landsitze, auf einer romantischen Anliöhe, den s. g. Mrichaelsberge, der mit den beiden, in nürdlicher Richtung gelegenen Hügeln, dem grossen und kleinen Spiellerge, nicht nur durch nalïrliche Verhälınisse, sundern auch durch küslliche Rebenplanzungen in Verbindung, und in fast europäischem Rute sleht, stand ehedem eine, dem heiligen Michael geweihte, nunmehr zerstörle, Kapelle. Nunmehr feicrt eine Menge von Tausenden alljälrrlich um Michaelis, am Fusse der Anhühe, auf dem s. g. Bruhlwiesen, ein merkwïrdiges Volksfest, den (mit dem Jahrmarkte zusammenfallenden) WV urstmarkt, der dem öffentlichen Verkaufe von tausend und zehntausend Würsten der verschiedensten Gattungen den Namen und eine Art von Berühmtheit verdankt. Das bunteste Menschengewïhl auf der grossen, mit Buden aller Art übersäeten Fläche, die hunderifache Erschülterung der Luft durch Geschrei und Geklirre, durch das Geheul wilder Thiere und das Schmettern der Trompelen, durch uen Tanz und das Wogen aller Farben und Grössen, - da reicht kein Pinsel und 
keine Feder aus, das Bild des freiesten Naturlebens kühn nnd vielgestaltig zu beschreiben. Selbst der Griffel eines Fenimore Cooper, der diese Gegenden um ihrer Schönheit und historischen Merkwïrdigkeit willen besuchte, zeichnet nur ein schönes und deutungsvolles Gemälde, - aber nur einen Hauch der Wirklichkeit!

Gegen Nordwest, auf dem s. g. Kastanienberge, ragt uns die Heiden - oder Ringmauer, ein Denkmal untergegangener römischer Grösse und Macht, entgegen. Sie ist der Hebe und Wendepunkt eines Cooper'schen Romans, und durch die romantische Fassung, aus der sie bestelit, auggezeichnet. -

An der südwestlichen Seite dieser Ringmauer befindet sich eine Oeffnung, welche dem Auge das entzückendste Panorama vorführt.

Vor uns, auf der mitlen im Dürkheimer Thale emporsleigenden Anhöhe, prangen die Ruinen der Abtey Lim$b u r g,-z u r$ Rechten breitet sich die lieblichste Wiesen Anlage aus, mit dem Spiegel eines sorglos sich durchschlängelnden Bachs, im Hintergrunde schimmert uns ein Theil der sonst mächtig-stolzen Flartenburg entgegen; an den Fuss uns'rer Anböhe lehnt sich in zerstreuten Gruppen das Dorf Grethen; ge'n Süden, im Dunkel des Waldes, leuchtet die feyerlich mahnende Kuppel der Seebacher Klosterkirche, noch ferner der alterthümliche Haupthurm der Burg zu IFachenheim. Die Blicke reichen ferner über die Stadt Dürkheim und unermessliche gold'ne Saaten hinaus bis an den Gebirgssaum, der in weitester Ferne den Horizont begränzt.

Der Nordseile der Ringmauer gegenüber erhebt sich auf einem Hügel der Teufelsfels, gewöhnlicher Teufelsstein genannt, den nach der Sage der Teufel selbst hieher versetzt, und dem er mit seinen Krallen ein ewiges, noch heule sichtbares, Denkzeichen eingedruckt habe. Noch weiter ge'n Nordwest erhebt sich bis zur Höhe von $1400^{\prime}$ über der Fläche 
des adriatischen Meerbusens der Peterskopf. Da ist kein Punkt der ganzen Umgebung, der schauerlich - schönere Bilder gewährte. Du stehst in einem chaotischen Felsenlager; zur I.inken erstarit Dein Auge vor dem Künige des ganzen Gebirgszugs, dem Donnersberge. Rechts dehnen sich die Berge zwischen Rüdesheim und Bingen, angelehnt an die nächtlichen Höhen des Taunus-Gebirges; ilum schliesst sich der Odinswald, und diesem der Schwarzwald an mit seinen schwarzgrünen Kuppen. Und inmilten dieses herrlichen Kranzes̀ die gesegnetsien Fluren der Pfalz, die besäet mit Słädten und Dörfern, vom Rbeine und zahlreichen Flüssen und Bächen bewässert, und durch eine fast italische Sonne gewärmt und beleuchtel!

Westwärts von dit'sem Punkte entsteigt dem Urgebirge der Heidenfels. Hier angelangt, blickst Du in die Schauer eines romantischen Abgrunds. Noch westlicher ruhet der Runfels, in weiterer Ferne der Flochberis, - tief unten aber weckt Dich die Hartenburg aus deiner träumerischen Begeisterung, und deren graues verwitterndes Gestirn mahnt Dich an die Vergänglichkeit der irdischen Pracht und Grösse!

Eine halbe Slunde endlich siidwestlich von Dürkheim, lächelt den Bewuhnern der Sladt einer ihrer belieblesten Vergnïgungsorte, das Dorf Seebach mit der Ruine einer einst schönen Klosterkirche, entgegen.

Ich könnte den Leser noch zu manchem Vergnügungs. platze in der Nachbarschaft Dürkheims geleiten; doch es ist Zeit, noch einen Augenblick bei dem romantischen Thale zu verweilen, dem uns're Sladt ihren Namen verliehen, um uns sodann dem Ernste wissenschaftlicher Betrachlungen hinzugeben.

Hinter Dürkheim eröfnet das wunderreiche Thal zunächst das schon bekannte Dorf Grethen. Die fernsten Häuser dieses sehr zerstreuten Weilers sind am Fusse eines inmit- 
ten des Thales aufsteigenden Berges gelagert. Keine Krone ziert schüner, als die Krone dieses Berges, die einst berühmle und gefürchlete Abtey Limburg, mit ihrem schlanken Thurme und ihrem umfangreichen, jetzt bemoosten Gemäuer, mit ihren einstigen Juwelen und Fresko-Gemälden, mit ihren Grüften und Chören! Mit dem Ruhme, der Silz und Schirm mächtiger Herzoğe und Prälaten, - gelehrter Bildung und kriegerischen Getümmels gewesen zu seyn !

An der nordöstlichen Seite des Limburger Berges liegen die Herzogsmühle und der Herzogsweiher, noch ferner das Weiler Hausen, einst die Stätte eines BenediktinerKlosters. Dann krümmt sich, in noch grösserer Entfernung, das Thal, und der Fuss des Wanderers erreicht die riesigen, kalten Mauern der Hartenburg.

Die Ruinen von Limburg und Hactenburg bilden die imposantesten Zierden dieses schauerlichschönen Thales. Von ihren Zinnen herab schweift das Auge über lachende Wiesengründe und gastfreundliche Häuser und $W$ ege zu den nahen, felsigen, mit dunkelm Nadelholze bewachsenen, Gebirgswänden hiniiber, und es ist Einem, als kehrte die alte, prunkende Ritter - und Minnezeit wieder, so heimisch und doch so öde! - fühlt man sich innerhalb der kahlen, epheubekränzten Mauern dieser luftigen Burgen, deren fürstliche Bewohner nächtlichen Eulen gewichen sind!

Verlassen wir nun die Burg, so gelangen wir heute zu dem Dorfe gleichen Namens. Wer erblickt nicht in diesem Wechsel der Dinge den Wechsel des Charakters der Zeit? - einst das Getümmel und der eherne Glanz der Ritter und Reisigen, - jetzt der häusliche Friede und Wohlstand eines Dorfs! -

Das Thal erstreckt sich noch weiter in der angegebenen Richtung; aber wir haben seine Juwelen gesehen, und wollen daher die Rückkehr zu der ruhig sprudelnden Quelle, 
von welcher wir ausgegangen sind, nicht zu lange verzögern.

\section{Geognostische und naturhistorische Verhältnisse der Umgegend Dürkheims *).}

Das Städtchen Dürkheim liegt am Fusse eines Lagers von Grobkalk (tertiären Kalkstein), welches vorı Deidesheim, 3 Stunden von Dürkheim, beginnt, und sich bis über Grünstadt auf der westlichen und nordwestlichen Seile von Dürkheim erstreckt. Dasselbe ist hie und da mit Adern von Kalkspath durchzogen, und geht bei Leisiadt an wenigen Stellen in Oolith und. Mergel aus. Dieses Lager von Grobkalk ist oberhalb Dürkheims nur etwa zeln Minuten breit, dehnt sich aber unterhalb desselben über $\frac{\mathrm{I}}{2}$ Stunde aus, und führt mehrere Versteinerunger. Es ist an bunten Sandstein, jene Gebirgsart, welche fast die ganze Mitte und Höhe des Rheinkreises, beinahe díe Hälfle des Kreisgebiets, umfasst, angelehnt. Unterhalb Dürkheim, in östlicher Richlung, besteht der Boden aus Mergelsand, Thon u.

*) Die geognostischen Angaben über die Ungegend im Allgemeinen, und die Quelle im Besondern, sind nach gütigsten Mittheilungen der ausgezeichnet kenntnissreichen Herren: Dom-Kapitular Wurschmitt in Speyer, und Salinen-Inspektor v. Schelf in Dürkheim, - die botanischen Verzeichnisse aber theils nach den Eröffnungen mehrer Freunde, theils nach $P$ etif's "Enumeratio plantarum in ditione florae palatinatus sponte crescentium; Biponti, apud Ritter, 1830. 8o", und endlich-z. Th. nach Pflanzen-Exemplaren aus der Umgegend Dürkheims, welche sich in meinen Herbarien befinden; bearbeitet, - Für die gewährten gütigen Mittheilungen hiemit öffentlich den gefühltesten Davk!

H. 
s. f., und gegen Süden, bis Meckenheim, u. s. f. aus Gerölle.

Die schnelle Verwitterung dieses bunten Sandsteingebildes trägt u. a. auch die Schuld, dass die Thalsohle in der Nähe des Hauptgebirgszugs grossentheils mit Gerölle (aus eben diesem bunten Sandstein gebildet) tief ausgefïllt ist. Dieses Gerölle nimmt in grösserer Teufe den Charakter eines aus eckigen und scharfkantigen, mit plastischem Thone zu einer gleichsam homogenen Nasse zusammengekneteten Conglomerates an. Der bunte Sandstein fïhrt an einigen Stellen etwas Schwefelkies, welcher mitunter in Brauneisenstein ïbergeht. Von Versteinerungen bielet er fast nichts dar, birgt dafür in seinen Ablösungen und Spaltungsklüften einen grossen Wasserreichlhum. In ihm steigen auch die Philippshaller Soolquellen anf, welche durch Bolurlöcher in nicht weniger als $30 o-9$ co' Tiefe erschreten worden sind. Ob. gleich dieses Einbrechen derselben im bunten Sandsteine auch anderswo als keirce neue Erscheinung gellen kann, und die relative Armuth derselben sich überall kund gegeben hat, so bleibt hier - im Vorübergehen bemerkt - nichts desto weniger das Emporsteigen des Quellenzullusses aus nambaften Teufen, die unbedeutende Veränderung in dem chemischen Bestande der genaunten Soolquellen zu allen Zeit - Perioden, die Steligkeit ihrer Temperatur von $+11^{\circ}-14^{\circ} \mathrm{R}$.; so wie endlich die periodische Schwefelwasserstoffocs - Exhalation und Ausscheidung won Eisenoxydhydrat bemerkenswerth.

Es vereinigen sich übrigens mehre Gründe für die Wahrscheinlichkeit der Annahme, dass unter dem bemerkten Kalkgebirge ein Lager von Gyps sich befinde. Auch die Salzquelle von Philippshalle giebt dieser Vermuthung Raum.

Was aber noch besondere Aufmerksamkeit verdient, ist der Umstand, dass in einer Entfernung von ${ }_{4}^{3}$ Slunden ein 
Basaltkegel, der s. g. Pechsteinberg in Forst, sich befirdet. Wir dürfen annehmen, dass derselbe uns zu dem Schlusse berechligt, mit den Vulkanisten auf frïhere, vielleicht selbst jetzt noch nicht erloschene, vulkanische Thätigkeit in, möglicher Weise grosser, Erdtiefe zu schliessen, und auch aus diesem Grunde dürfte die Nähe von Gyps unter dem Kalklager zu vermuthen seyn, so wie derselbe Grund auf die Existenz von Schwefelkies in dem Sandsteingebilde hinweist.

In naturhistorischer Beziehung (im engern Sinne) vermag ich keine vollständigere Mittheilungen zu machen.

Unter den seltnern phanerogramischen Gewächsen sey es erlaubt, als solche, die der Umgegend Dürkheims bis auf eine Entfernung ron zwei bis drei Stunden angehören, folgende aufzuführen:

$$
\text { Najades. }
$$

Hippuris vulgaris $\mathbf{L}$.

Caulinia fragilis W.

Zanichellia palustris Michx.

Myriophyllum spicatum L.

Potamogeton natans L. Var.

angustatus $M$. et $K$., et Var.

intermedius $M$. et $\mathrm{K}$.

- crispus $\mathbf{L}$.

- acutifolius M. et $K$.

- pusillus L.

Aroideae.

Arum vulgare La m.

Acorus Calamus L.

Sparganium ramosum $\mathbf{R}$.

Typha latifolia $\mathbf{L}$.

- angustifolia $\mathbf{L}$.

Gyperoideae.

Carex intermedia Good.

- Schreberi W.

- vulpina $\mathbf{L}$.
Carex muricata. Var. Longibracteata $S p r$.

- teretuiscula Good.

- clandestina Good.

- digitata $\mathbf{L}$.

- tomentosa $\mathbf{L}$.

- supina Wahlb.

- distans $L$.

- limosa L.

- Pseudocyperus L.

- pendula Huds.

- riparia Curt.

- hirta Var. hirtaeformis Pers.

Scirpus uniglumis Link.

- ovatus $R$.

- acicularis $\mathbf{L}$.

- Baeothryon Ehrh.

- lacustris $\mathbf{L}$.

- Tabemaemontani $\mathbf{L}$.

- maritimus L. 
Scirpus compressus $\mathrm{Pers.}$

Schoenus nigricans $L$.

Eriophorum vaginatum $\mathbf{L}$.

Cyperus fuscus $\mathbf{L}$.

Gramineae.

Spinia alba Schrad. Var. decumbens $G$ a ud et Var. patula $G$ a ud.

Panicum verticillatum $L$.

Syntherisma ciliare S chrad.

Stipa pennata $\mathbf{L}$.

- capillata $\mathbf{L}$.

Avena tenuis M.

Bromus triflorus flavescens $P$.

- pratensis L.

Arundo sylvatica Schrad.

Festuca Myurus L.

- conamunis. Var. duriuscula $P_{\text {. }}$, nemoralis M. et $K$.

- arundinacea Schreb.

- sylvatica Vill.

- gigantea Vill.

- aspera M. et K.

- inermis DC.

Bromus decipiens. Var. patulus $\mathrm{M}$. et $\mathrm{K}$.

Glyceria distans Wahl.

- aquatica Pressl.

Dactylis glomerata $\mathbf{L}$.

Koeleria cristata Pers.

Sesleria coerulea Ard.

Melica ciliata $\mathbf{L}$.

- nutans L.

- uniflora Retz.

Triticum repens, Var. arenosum $\mathbf{L}$.

- caninum Schreb.
Lolium arvense $\mathbf{L}$.

Nardus stricta $\mathbf{L}$.

Andropogon Ischaemum $\mathrm{L}$.

Junceae.

Juncus squarrosus $\mathbf{L}$.

- bottnicus Wahlb.

- capitatus Weig.

- fusco-ater Schreb.

- lampocarpus Ehrh.

Asparageae.

Convallaria multiflora $\boldsymbol{L}$.

- verticillata $\mathbf{L}$.

Anthericum Liliago $\mathbf{L}$.

- ramosum $\mathbf{L}$.

Asparagus officinalis $\mathbf{L}$.

Liliaceac.

Lilium Martagon L.

Muscari racemosum Miller.

- comosum Miller.

- botryoides Miller.

Ornithngalum bohemicum. Var. saxatile Koch.

Allium acutangulum $\mathrm{W}$.

- rotundum $\mathbf{L}$.

- oleraceum $\mathbf{L}$.

- sphaerocephalum $\mathbf{L}$.

Tofieldia calyculata IV ahlb.

Alismaceae.

Triglochin palustre L.

- maritimum $\mathbf{L}$.

Alisma Plantago L. Var. angustifolium.

Hydrocharis Morsus ranae $\mathbf{L}$.

Sagittaxia sagittifolia $\mathbf{L}$.

Butomus umbellatus $\mathbf{L}$.

Nymphaea alba $\mathbf{L}$.

Trapa natans $\mathbf{L}$.

Arch.d. Pharn. II. Reire. VII. Bds.1.Hft. 


$$
\text { Irideae. }
$$

Iris germanica $\mathbf{L}$.

- sibirica $L$.

Orchideae.

Orchis coriophora $\mathbf{I}$.

- ustulata $\mathrm{L}$.

- militaris. Var. fusca Iacq. et galeata $\mathbf{L}$ am.

- hircina Scop.

- sambucina L. Var. incarnata.

- odoratissima $\mathbf{L}$.

- viridis $\mathbf{L}$.

Herminium Monorchis R. Br.

Ophrys myodes R. Br.

- apifera R. Br.

Neottia spiralis $\mathrm{S} w$.

- repens $S w$.

Epipactis palustris $S w$.

- latifolia $S w$.

Distomea pidus avis Spenner.

- ovata $S$ p.

Cephalanthera pallens $\mathrm{Rich}$.

- rubra Rich.

Coniferae.

Pinus Picea $\mathbf{L}$.

Juniperus communis $\mathbf{L}$.

Amentaceae.

Salix amygdalina $L$.

- purpurea L.

- rubra Huds.

- aurita $\mathbf{L}$.

- repens L. Var. parrifolia $\mathrm{Sm}$.

Quercus sessiliflora Sm.

- pedunculata $W$.

Ulmus campestris $\mathbf{L}$.
Urticeae.

Urtica. Dodartii L.

Parietaria erecta $M$. et $K$.

Tricoccae.

Euphorbia falcata L.

- Gerardiana Jacq.

- palustris L.

Mercurialis perennis $\mathbf{L}$.

Aristolochiae.

Aristolochia Clematitis $L$.

Elaeagneae.

Thesium moutanum L.

- intermedium Schrad.

- pratense Ehrh.

- alpinum $\mathbf{L}$. Thymeleae.

Daphne Mezereum $\mathbf{L}$.

- Cneorum L.

Stellera passerina $\mathbf{L}$. Polygoneae.

Polygonum minus $L$.

- Hydropiper $\boldsymbol{L}$.

Rumex crispus. Var. unigranis $\mathbf{L}$.

- obtusifolius $\mathbf{L}$.

- Hydrolapathum $\mathbf{H}$ uds.

- scutatus $\mathbf{L}$.

Chenopodieae.

Chenopodium intermedium $\mathrm{M}$. et $\mathrm{K}$.

- leiospermum DC. Var. opulifolium S chrad.

- ficifolium Sm.

- glaucum $\mathbf{L}$.

- polyspermum. Var. racemosum $\mathbf{L}$.

Atriplex patula $\mathbf{L}$.

- angustifolia Sm. 
Atriplex oblongifolia M. et $K$.

Polycnemum arrense $I_{\text {. }}$

Amaranthaceae.

Amaranthus Blitum I.

Plantagineae.

Plantago arenaria M. et $\mathrm{K}$.

Primulaceae.

Androsace maxima $\mathrm{L}$.

Lysimachia nemorum $L$.

Hottonia palustris $\mathbf{L}$.

Samolus Valerandi L.

Lyndernia pyxidaria.

Glaux maritima $\mathbf{L}$.

Utricularieae Link. Utricularia vulgaris $\mathbf{L}$.

- minor $L$.

Scrophularineae. Digitalis ochroleuca $\mathbf{J}$ a cq. Antirchinum majus $\mathbf{L}$.

- Orontium $\mathbf{L}$.

Rhinanthaceae.

Veronica montana $\mathbf{L}$.

- Teucrium. Var. latifolium min. Schrad. - prostratum $\mathbf{L}$, angustifol. Schmidtii $R$. et $\mathrm{Sch}$.

Euphrasia lutea L.

Orobancheae.

Orobanche elatior Sutton.

- arenaria Borkh.

- ramosa $\mathbf{L}$.

Lathraea squammaria $L$.

Jasmineae.

Fraxinus excelsior.
Labiatae.

Salvia pratensis, Var. parviflora $\mathrm{L}$.

Teucrium Botrys L.

- Scordium L.

Betonica stricta Ait.

Stachys Sideritis Vill.

Leonurus Cardiaca $\mathbf{C}$.

Melissa Calamintha $\mathbf{H}$.

Prunella laciniata $\mathbf{L}$.

Asperifoliae.

Myosotis intermedia Link.

Echinospermum Luppula L els$\operatorname{man} n$.

Cynoglossum montanum Lam.

Lythospermum purpureo-coeruleum $\mathbf{L}$.

Pulmonaria angustifolia $\mathbf{L}$.

Heliotropinm europaeum $\mathbf{L}$.

Solaneae.

Plhysalis Alkekengi L.

Atropa Belladonna L.

Datura Stramonium L.

Verbascum officinarum. Var.

Thapsus L.et Var. phlomoides $L$.

- Lychnitis. L. Var. albiflorum $L$.

Gentianeae.

Gentiana cruciata $L$. utriculosa $\mathrm{L}$.

Chlora perfoliata Adans.

- serotina Koch.

Villarsia nymphoeoides Vent.

Ericaceae.

Arbutus Uva Ursi L.

Pyrola minor $\mathrm{L}$.

$3 *$ 
Vaccinium uliginosum $\mathrm{L}$.

Rubiaceae.

Galium saccharatum Scop.

- agreste Scop.

- gracile Wallr. Var. anglicum $\mathrm{Huds}$.

- boreale Wallr.

- sylvaticum $L$.

- sylvestre L.

Asperula odorata L.

- arvensis $\mathbf{L}$.

- galioides Bbst. Caprifoliaceae.

Lonicera Gaprifolium L,

- Xylosteum L.

Viburnum Lantana $L$.

Sambucus Ebulus L.

- racemosa $L$.

Rhamneae.

Rhamnus catharticus $L$.

Ilex aquifolium $L$.

Grossularieae.

Ribes alpinum $L$.

Berberideae.

Berberis vulgaris $L$.

Acerineae.

Acer pseudoplatanus $L$.

- platanoides $\mathrm{L}$.

- monspessulanum L.

Cistineae.

Helianthemum fumana Desf. Polygaleae.

Polygala polymorpha. V ar.uliginosa $R$ eichb. et $\mathrm{Var}$. serpyllacea Weihe.

Rutaceae.

Dictamnus fraxinella Pers.
Monotropa hipopitys L.

Capparideae.

Reseda luteola $L$.

Drosera rotundifolia L.

- obovata M. et K.

Hypericineae.

Hypericum montanum $\mathbf{L}$.

- hirsutum L.

- pulchrum L.

Campanulaceae.

Jasione perennis $\mathrm{L}$.

Campanula persicifolia $L$.

- glomerata L.

- Prismatocarpos L. Valerianeae.

Fedia dentata Vahl.

Aggregatae.

Dipsacus laciniatus $L$.

Compositae.

Carduus acanthoides L. - crispus L.

Cirsium Allionii $\mathrm{L}$.

Serratula Pollichii DC.

Arctium majus Thuill.

Centaurea nontana DC.

- panicula $L$.

- Jacaea Var. transyl-

vanica $\mathrm{W}$.

Chrysocoma Linosyris L.

Artemisia pontica L.

Conyza squarrosa $L$.

Arnica montana $L$.

Inula britannica $L$.

- Pulicaria L.

- germanica $L$.

- hirta L.

Erigeror acre Var. alpinum $L$.

Aster amellojdes L. 
Aster salignus W.

Senecio erucaefolius $L$.

- ovatus I.

Anthemis tinctoria $\mathbf{L}$.

Calendula arvensis $L$.

Thrincia hirta R.

Crepis paludosa Mönch.

- tectorum Wallr.

Barkhausia foetida $M \ddot{n} n \mathrm{ch}$.

Hieracium procerumV ar. praealtum Vill. et Var. Auricula W.

Lactuca virosa $L$.

- saligna L.

- perennis L.

Prenanthes purpurea L.

Chondrilla juncea L. et Var. latifolia L.

- muralis Lam.

Hypochaeris glabra L.

Podospermun purpureum K. et

$\mathrm{z}$.

Lapsana minima All.

Plumbagineae.

Armeria vulgaris $\mathrm{W}$.

Umbellatae.

Bupleurum rotundifolium L.

- tenuissimum L.

Trinia Henningia B bst.

Seseli annuum L.

Carum Bulbocastanum Ko.ch.

Oenauthe Lachenatii $V$ ar. pimpinelloides $P$. et peucedanifolia $P$.

Apium graveolens $\mathbf{L}$.

Platyspermum grandifiorum M. et K.

Caucalis latifolia L.
Scandix Pecten L.

Cerefolium vulgaris Pers.

Sium latifolium $L$.

Helassidium nodiflorum M. et K.

$$
\text { - repens } \mathrm{Koch} \text {. }
$$

Peucedanum alsaticum $L$.

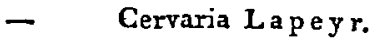

- OreoselinumMönch. Saxifrageae.

Adoxa moschatellina L. Onagrarieae.

Epilobium grandiflorum All.

- spicatum Lam.

Circaea alpina L.

Salicarieae.

Salicaria hyssopifolia L.

Cruciferae.

Arabis auriculata Lam.

Turritis glabra $\mathbf{L}$.

Barbarea Linnaei R.Br.

Erysimum orientale Spenner.

Cheiranthus Cheiri L.

Napus Villarsii.

Cardamine micrantha $\mathrm{Sp}$.

- impatiens L.

Cochlearia Armoracia L.

Thlaspi montanum L.

Sennebiera Coronopus $L$.

Iberis amara $\mathrm{L}$.

Hutchinsia petraea A it.

Rapistrum rugosum B e rg.

Papaveraceae.

Actaea spicata.

Fumariaceae.

Corydalis Halleri DC.

Ranunculaceae.

Thalictrum montanum $V$ ar. pu- 
bescens Schleich, et

Var. saxatile.

Thalictrum galioides $\mathrm{N}$ estler. Adonis aestivalis $\mathrm{L}$.

- flammea $\mathbf{J a c q}$.

- vernalis $\mathbf{L}$.

Ranunculus Lingua $L$.

- aconitifolius $\mathbf{L}$.

- Philonotis L.

- parvulus $\mathbf{L}$.

- polyantliemos Var.

latifolius.

- bydrocharis DC.

Anemone sylvestris L.

- xanunculoides L.

Hepatica triloba Vill.

Helleborus foetidus $\mathbf{L}$.

Nigella arvensis $\mathrm{L}$.

Auqilegia vulgaris $\mathrm{L}$.

Aconitum Lycoctonum L.

Papilionaceae.

Trifolium hybridum $\mathrm{L}$.

- rubens L.

- ochroleucum L.

- filiforme $\mathbf{L}$.

Melilotus officinalis W.

- IeucanthaK och.

Medicago falcata $\mathbf{L}$.

- minima Lam.

Lotus siliquosus L.

Astragalus glyciphyllos L.

Lathyrus Aphaca L.

Vicia pisiformis $\mathrm{L}$.

- cassubica $L$.

- lathyroides L.

Hippocrepis comosa L.

Orobus niger $\mathrm{L}$.

- tuberosus L.
Geraniaceae.

Geranium pratense $\mathbf{L}$.

- pyrenaicum L.

Oxalis stricta $\mathrm{L}$.

Impatiens noli tangere $L$.

Malvaceae.

Althaea officinalis $\mathbf{L}$.

Violarieae.

Viola palustris $L$.

- arenaria DG.

- stagnina $\mathrm{Kit}$.

Caryophylleae.

Silene Otites L.

- conica $\mathrm{L}$.

- inflata Var. alpina L am.

Agrostemma coronaria $\mathrm{L}$.

Arenaria marina L.

- fasciculata L.

Montia fontana Var. fluctans

Michel.

Paronychieae.

Herniaria hirsuta L.

Sedeae.

Sedum sexangulare $L$.

- villnsum $\mathbf{L}$.

Sempervivum hirtum L.

- Rosaceae.

Rosa spinosissima L. et Var. mitissima $\mathrm{Gm}$.

- collina Schimp. (PIur.

Variet.).

- glandulosa Schimp. (Plur. Variet.).

- gallica $L$.

Alchemilla vulgaris Var. hirsuta $L$.

Potentilita fragaria Poir. Rubus Idaeus $\mathbf{L}$. 
Rubus polymorphusVar, arvensis flor.Friburg.

Pyrus communis $\mathrm{L}$.

- Malus L.

- Aria Ehrh.

- torminalis Ehrh.

Mespilus germanica $L$.

Cerasus Padus DG.

- acidus Borkh.

Lorantheae.

Viscum album. L.

Dieser Pflanzenreichthum (wir haben, wie gesagt, die gewöhnlichern ganz übergangen) darf an einer so submontanen und fruchtbaren Gegend, wie jene Dürkheims, nicht überraschen. - Auch in ory kt og nostischer Beziehung bietet Dürkheim manches Beachtenswerthe dar, woriiber ich indessen, aus Mangel an Lokalkenntniss, keine genügenden Nachweisungen zu geben vermag. - Endlich soll sich jene Gegend in entomologischer Beziehung vorzïglich auszeichnen. -

\section{Ursprung und nächste Umgebung der Quelle.}

Wir wenden uns nunmehr zur Betrachtung der Quelle, deren Ursprung in der Nähe von Dürkheim wir bereits. angedeutet haben *).

Sie entspringt nämlich in dem berührten Sandstein-Gerölle in einer Tiefe von $16^{\prime}$, und wurde durch Nachgrabung erschroten. Die obere Decke bestand aus einer $3^{\prime}$ mächligen Dammerile-Schicht, und diese wurde von einer 13' mïchtigen Torfschicht unterteuft, unter welcher die Gerölllage (vergl. den vorigen Abschnitt) entblösst wurde, aus welcher die Quelle aufsteigt. Sie wurde später, nach gewölnnlicher Brunnenart, mit Sandsteinschaalen in Cylin-

*) Die hieher gehörigen Mittheilungen erhielt ich durch die Güte des Herrn v. Schelf, nachdem die Analyse des Wassers schon lange beendigt war. Die hier oben angedeuteten geognostischen VerhäItnisse beleuchten die Resultate der chemischen Zerlegung za meinem Vergnïgen auf eine damit sehr übereinstimmende Weise.

H. 
derform ausgekleidet, und Letztere auf einen Schellenrost geselzt. - Sie wird durch eine gewöhnliche Handpumpe zur Benutzung gebracht. -

Auf längere Zeit in Ruhe gelassen, sleigt die Quelle nicht über das Ausmündungs-Niveau. Da nun der Fassungsraum dieser Quelle einen Durchmesser von $4^{\prime}$ behauptet, welcher noch überdies $16^{\prime}$ tief ist, so dürfte das niemalige Uebersteigen des Zuflusses mit Grund der nicht wasserdiclten Fassung zuzuschreiben seyn. Es dürfte daher auch der ursprüngliche eigenthümliche Gehalt des Wassers an fremden Bestandtheilen noch in Frage gestellt werden künnen, weil zu vermuthen steht, dass zwischen dem Wasser der Quelle und jenem des vorüberfliessenden Bachs irgend eine Communication Stalt finde; deun es ist nicht wohl abzuschen, wie der hydrostatische Druck dieser nicht bedeutend hohen Wassersäule in Stande seyn sollte, das Aufsteigen derselben zu erschweren. - Um indessen diese, und so manche andere sich hieran reihende Fragen entscheidend zu beantworien, bedarf es länger andauernder Gewältigungs-Versuche und periodischer chemisch-analytischer Prüfungen des Wassers.

Da der steinerne Verschluss und die Pumpen - Vorrichtung das Aufsteigen des Wassers nicht beobachten lassen, so kann auch der Grad und die Art der Gas-Entwicklung daselbst nicht wohl wabrgenommen werden. Inzwischen ist der Schwefelwasserstoffgeruch in der den Brunnen zunächšt umgebenden Atmosphäre nur schwach, und es ist mir wahrscheinlich, dass diese Luft-Entwicklung als das Resultat der gegenseitigen Zersetzung des im bunten Sandstein enthaltenen Schwefelkieses und der in Verwesung übergegangenen Pflanzenstoffe der Moordecke zu betrachten ist. Es wäre übrigens von Interesse, die Gas-Entwicklung an Ort und Stelle selbst wahrnehmen, und ihre Natur, besoriders 
rücksichllich etwaiger Kohlenwasserstoff - *) und Phosphorwasserstoff-Beimischung untersuchen zu können.

Herr Oberle will übrigens eine periodisch-stärkere, und alsdann die Geruchs-Organe heftiger afficirende Gas Entwicklung wahrgenommen haben, - eine Erfahrung, die auch bei den Soolquellen vorgekommen ist, und an deren Eintritt der Wechsel der Temperatur - und Witterungs Verbältnisse Theil zu nehmen scheint, Diese periodische Gas-Fluctuation ist bereits an mehreren andern Quellen beobachtet worden, und verdiente mehrseitige gründlicle Nachforschung, da die Summe aller bedingenden Momente unstreilig noch nicht gekannt ist.

Obgleich übrigens diese Quelle von der einen Soolquelle nur 120 Fuss, von der andern 53o Fuss entfernt liegt, so ist ein wechselseitiger unlerirdischer Verband derselben doch um so mehr zu bezweifeln, als der grosse Teufen-Unterschied dieser Ansicht entgegensteht.

Aus einer Reihe von zehn angestellten Gewältigungs Versuchen ergieb̆t sich im Mittel eine Zufluss - Quanlität von 1o Bayer'schen Cubikfussen in der Minute; dies betrïgt für die Stunde 240 Cubikfuss, und für den ganzen Tag 5760 solcher Cubikfusse.

Reducirt man diese Quantität auf französisches Maass (den Litre zn 5o,4124 Par. Cub. Zollen), so berechnet sich ohiger Zufluss für die Minule auf 102,6264; für die Slunde auf 6157,5840 ; und für den Tag auf 147782,016 Lilres, oder auf 147,7820 Fuder (Kilolitres).

Von der Pumpe weg läuft das Wasser in kupfernen Rübren unmiltelbar in den Wärmekessel, und mittels gleicher Leitungs-Vorrichlung in die an die Quelle anstossen-

*) Sumpfluft. -

H. 
den Bad - Cabinelte, kann aber auch unter das Dachgebälke zum Bewahren in das vorgerichtete Reservoir gegeben werden.

Ausscheidung von Eisenoxyd im geschöpften Wasser ist bisher noch nicht beobachtet worden.

Es ist hier der Ort, zu bemerken, dass in ziemlich beträchtlicher Entfernung von der Oberle'schen Quelle noch eine andere, die s. g. Brï̈lenquelle zu Tage kömmt, die durch starken hepatischen Geschmack und bedeutenden Eisenoxydhydrat-Absalz grössere Reichlalligkeit verkïndet, und somit den Schwefel-Stahlwässern anzugehören scheint. Ich hoffe, in der Folge mehr ïber diese Quelle millheilen zu können. Sie ist übrigens von der OberJe'schen Quelle so weit entfernt, dass sie mindestens in keinem oberirdischen Zusammenhange mit ihr zu stehen scheint.

\section{Untersuchung des Oberle'schen Heilwassers.}

Ich erhielt dieses Wasser in mehreren sorgfältig verpichten steinzeugenen, Selterserwasser - Krügen eingeschlossen, mit der Versicherung, dass auf die Füllung dieser Flaschen alle $\Lambda$ ufmerksamkeit verwendet worden sey. Es ist also zu erwarten, dass die Ergebnisse der unten folgenden Analyse rücksichtlich der fixen Bestandibeile auch dann wenig $\Lambda$ bweichungen dargeboten haben würde, wenn die Untersuchung an der Quelle vorgenommen worden wäre. Ob inzwischen die jetzige Fassung der Quelle den Zudrang fremdartigen Wassers, und mit ihm alle und jede Modification oder Schwächung des chemischen Mischungs-Verhälınisses abwehre, dürfte nach den Aeusserungen des Herrn v. Schelf zu bezweifeln seyn. In so ferne künnte man sich wohl zu der Hoffnung berechligt fühlen, den chemischen Gehalt, und mit ihm die therapeutische Wirksamkeit dieses Quellwassers einst noch gehoben zu sehen. 
Die gasförmigen Beslandtheile anlangend, so ist deren genaue Bestimmung, wie sie die Wissenschaft fordert, fast nur an der Quelle selbst möglich, es müssle denn die Füllung der Krüge unter ganz besondern, von Rose in der neuen Auflage seiner Handbuchs der analytischen Chemie angedeuteten Vorsichtsmaassregeln Statt finden. Glücklicher Weise aber gewährt die Bestimmung der gasigen Bestandtheile nur dann erbeblichren Nutzen, wenn deren qualitative oder quantitativen Verhältnisse einen wesentlichen Unterschied des Heilwassers von gewölnlichen Quellwässern begründen. Auf den Schwefelwasserstoff-Gehalt des yorliegenden Wassers gebe ich vor der Hand aus dem Grunde nicht viel, weil er jedenfalls, selbst in Ursprunge, unbedeutend, und der Verllüchtigung in so hohem Maasse fähig ist, dass ich nur am lnhalte einzelner der doch mit vieler Vorsicht gefüllten und bestens verschlossenen Krïge Spuren von hepatischem Geruche und Geschmacké wahrgenommen habe, weil dieser Gasgehalt also keine medicinische Bedeutsamkeit in sich trägt, und weil endiich, was auch aus dem unier II und III Gesagten mit Wahrscheinlichkeit erschlossen werden kann, keine Schwefelsalze im Wasser enthalten sind, wie die Analyse zeigen wird. Ja dieser Schwefelwasserstoff-Gehalt war in dem mir überschickten Wasser von der Art, dass er von keinem der empfindlichsten chemischen Reagentien angezeigt wurde.

Da nun, nach dem Empfange des Wassers, die Untersuchung desselben, um dringender Geschäfte willen, einige Tage verschoben werden musste, so lagerte icls die Krüge einstweilen, die Mündung abwärts gerichtet, auf Sand im Keller, um den Inlıalt derselben möglichst unverändert zu bewahren.

Wir gehen nun zur Betrachtung der angestellten Beobachlungen und Versuche, so wie der erhaltenen Resultate über. 
1. Physische Charaktere des Mineralwasero.

Farbe und Durchsichtigkeit.

In grössern Schichten zum Gelblichen sich neigend, in kleinern farblos, ganz durchsichtig.

Geruch.

An dem Inhalte einzelner (jedoch nicht aller) Krüge konnte ein sehr schwacher hepatischer Geruch wahrgenommen werden.

Geschmack.

Ei was eisenhaft, kaum - und wieder nur bei dem Wasser einzelner Krïge, hepatisch.

Es perlt

sehr wenig heim Herausgiessen.

Der Kochpunkt

slimmt mit dem des nicht luftfreien destillirten $W$ assers bis auf die letzten Decimalstellen überein.

Das specifische Gewicht

belrïgt, nach drei, bei gleicher Temperatur (+140 C.) vorgenommenen Wägungen 1,0011 .

Die Temperatur

es Wassers fand Hcrr v. Schelf an der Quelle selbst $=$ $+10^{\circ}$ C. $\left(+8^{\circ}\right.$ R. $)$.

2. Aufsuchung der chemischen Charaktere und Bestand theile.

Wir berücksiclıtigen

A. die fixen, und dann, als Anhang,

B. die gasigren Bestandtheile, und zwar:

$\Delta$. in a) qualilativer, und

b) quantitativer Beziehung.

$\mathrm{Zu} \mathrm{A.,} \mathrm{a.}$

a) Die Präliminar-Versuche bestanden in der Vintersuchung des Verhaltens des ungekochten wie des gelochten IFassers zu allgemeinen Reagentien, wie folgende Tabelle anschaulich machen wird. 


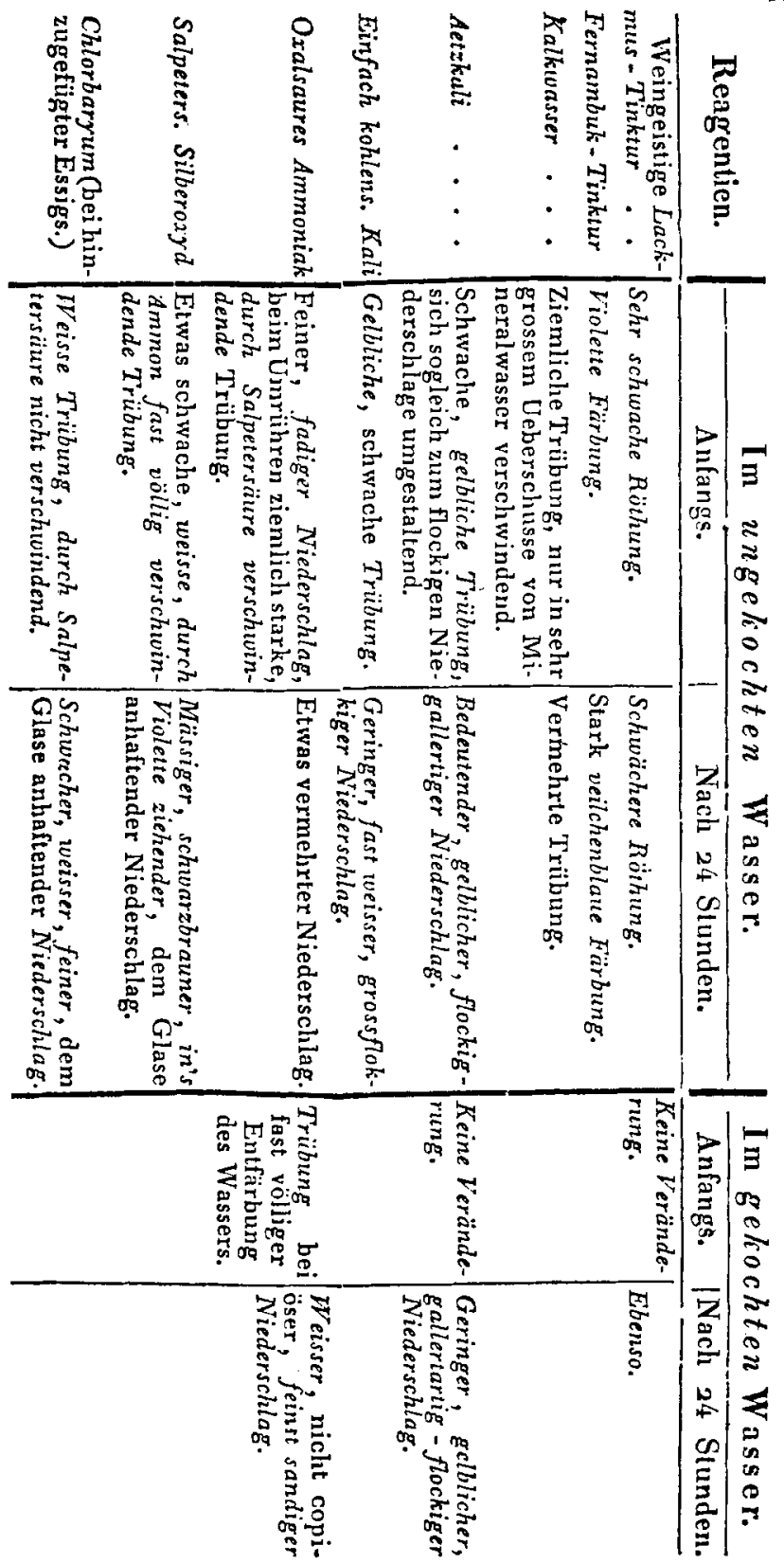


46

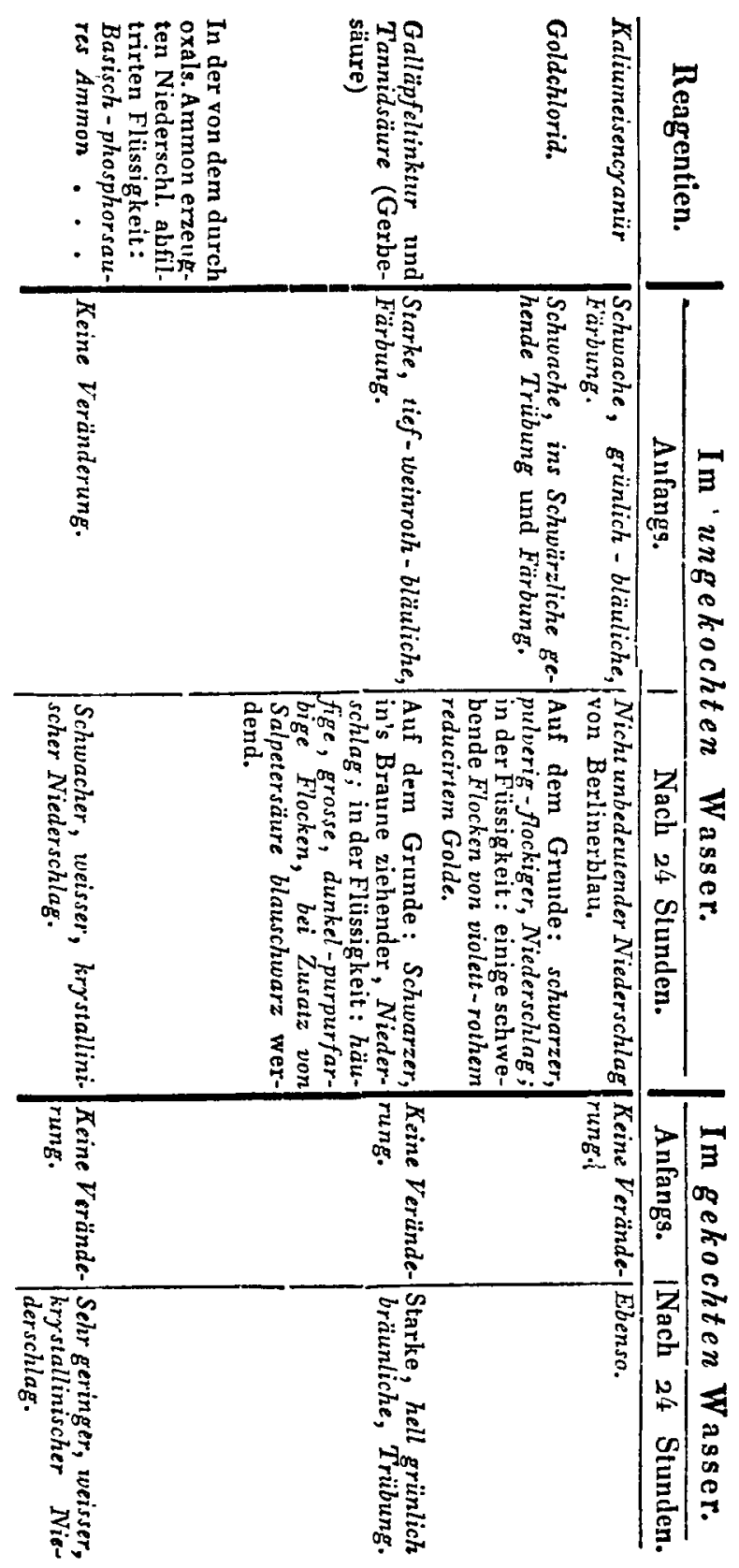


Als vorläufige Resultate dieser Präliminar - Versuche gehen herror:

Die Gegenwart blosser Spuren von Hydrothionsüure; ein geringer Gehalt an freier Kohlensäure und an Magnesia-Salzen; schwefelsaure und salzsaure Salze von Kalk, Magnesia und allenfalls auch Thonerde; endlich kohlensaures Eisenoxydul in nicht unbedeutender Menge, und ein organisches Princip. *).

$\beta$ ) Der, wenn auch sehr schwache hepatische Geruch und Geschmack des Wassers gaben Veranlassung, nach der Gegenwart einer Schwefelverbindung zu forschen. Aber weder schwefelsaures Kupferoxyd noch Kupferchlorid, brachten auch nur dunkle Färbung darin hervor. Jene z. Th. schwarzbraune Färbung des durch Silbernitrat erzeugten Niederschlags scheint also z. Th. durch die Gegenwart einer organischen Substanz vermiltelt worden zu seyn.

r) Behufs der Erforschung salpetersaurer Salze wurden einige Pfunde des Wassers vorsichtig zur Trocknissverdampfen gelassen. Der trockne Rückstand zeigte auf glühenden Kohlen und vor dem Lüthrohre keine Spur von Verpuffung. Auch durch einen Krystall von Eisenvitriol ward in der Lösung dieses Rückstands keine Salpetersïure angezeigt, nachrlem Schwefelsäure hinzugefiigt worden war.

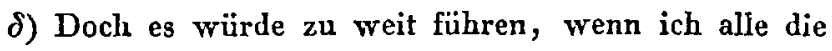
zahlreichen verneinend beantworteten Versuche, die ich angestellt habe, ausfiilurlich erzählen wrollte; denn als solche muss ich bezeichnen: die Prüfungen auf Jod - und BromGehalt, auf Lithion- Zink- Kupfer-Ammon-Strontianerde und Baryterde - Fluorcalcium - Thonerde-Phosphat-

*) Die Bildung eines eigentlichen Badsteins findet, nach einer brieflichen Mittheilung des Herrn Inspekfors S chelf, beim Kochen des Wassers in den metallenen Kesseln nicht Statt. 
Borsäure - Quell- und Quellsatzsäure-Brunnen- und Kanitzsäzure.

ह) Wohl aber liess sich, nach bekannien Methoden, die Gegenwart von geringen Mengen phosphorsaurer Kalkerde entdecken.

$$
\text { Zu A., b. }
$$

5) In einer porcellanenen Schaale wurden unter den erforderlichen Vorsichtsmaassregeln 400 Unzen des Mineralwassers langsam bis zur Trockne verdampfen gelassen. Bald schieden sich ockergelbe Flocken ab, und nachdem die Wassermenge bis auf ein Sechstheil ihres ursprünglichen Volumens zurücligefübrt war, begann auch die Absonderung einer krystallinischen Salzhaut. Der gesammte Rïickstand des Wassers endlich war erdig, von lichter Ockerfarbe, und sein Gewicht betrug 8,125 Grammen.

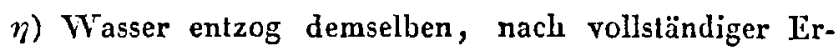
schöpfung 5,o5o Grm. Der völlig eingetrocknete Rï̈ckstand dieser wässrigen, wieder verdampften Lösung war ockerbräunlich, salzig, von spurweiser alkalischer Reaction. Dieses letztere Residuum möge, um der Verdeutlichkeit willen, mit $R^{\prime}$; der rom Wasser nicht aufgenommene Antheil aber mit $\mathrm{R}$ bezeichnet werden.

v) Der Rückstand $\mathrm{R}^{\prime}$ wurde wieder in Wasser gelöst, und die Lösung mit einigen Tropfen verdünnter Salpetersäure versetzt. Durch Barytnitrat wurde sodann alle im Wasser enthaltene Schwefelsäure als Barytsulfat gefällt, dessen Gewicht nach dem Trocknen 4,0 Grm. (= 1,3749 Grm. Schwefelsäure) betrug.

๖) Aus der vom schwefelsauren Baryt abfiltrirten Flüssigkeit ward durch Silbernitrat die ganze Menge des im Mineralwasser enthaltenen Chlors niedergeschlagen. Das erhaltene Silberchlorid wog nach dem Glühen 5,734 Grm. $(=1,41458$ Grm. Chlors). 
x) Nun schritt man, in soferne die Abwesenheit eines löslichen Phosphats in unserm Wasser bereits ausgemiltelt war, zur Bestimmung der Basen.

Die abfiltrirle, um der Vorsicht willen noch mit etwas Chlorammonium versetzte, Flüssigkeit ward mit Aetzammon vermischt, wodurch o,o 1 Grm. Thonerde niedergeschlagen wurde.

ג) Aus der davon durch Filtration geschiedenen Fliissigkeit ward der Kalk durch oxalsaures Ammon gefällt. Der Niederschlag gab nach dem Glühen, wobei auf die nülligen Vorsichtsmaassregeln Rücksicht genommen worden war, $0,052 \mathrm{Grm}$. kohlensauren Kalks (=0,029271 Grm. Kalks).

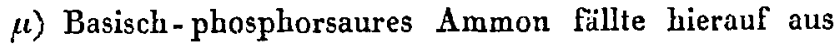
der wieder abfiltrirten Lösung die Magnesia. Der gehörig getrocknete Niederschlag wog 0,182 Grm. (=0,027852 Grm. Magnesia).

v) Die noch übrigen Flüssigkeiten wurden nunmehr eingedampft, der Rückstand scharf getrocknet, selbst schwach geglüht, ủann mit hydrochlorsäurehaltigem Wasser ausgezogen, wieder getrocknet und geglüht. Der sobehandelte Rïckstand wog 4,013 Grm. Er ward wieder in Wasser gelüst und mit alkoholischer Platinlösung zerlegt. Der nach zwölf Stunden abgelagerte krystallinische Niederschlag wog 0,207 Grm. (= $0,021 \mathrm{Grm}$. Kali's $=0,0327045 \mathrm{Grm}$. Chlorkaliums) wornach noch 3,9802945 Grm. Cblornatriums zu berechnen blieben.

Fassen wir nun die Resultate der bisherigen Versuche in Kürze zusammen, und berechnen wir die gefundenen Säuren und Basen nach dem, von den meisten Chemikern in Ermanglung beweisender Gegengründe, einmal angenommenen Grundsatze, dass die stärkere Base auch die stärkere Säure anziehe u. s. f. *).

*) Die Chemie hat rücksichtlich der Untersuchung der Art Arch. d.Pharm. II. Reihe. VII.Bds. 1. Hft. 
Gefundene Schwefelsäure . . . . . 1,3749 Grm. Hiervon für 0,02L Kali . . . 0,01783 Grm.

- $\quad 1,06623$ Natron (aus $1,74593 \mathrm{Na} \mathrm{Cl}_{2}$ ) . . . 1,35702 Grn.

Gefundenes Chlor . . . . . . . . . . $\frac{1,3749}{1,41458}$

Hievon für $0,052 \mathrm{Grm}$. $\dot{\mathrm{Ca}} \ddot{\mathrm{C}}=0,02927 \mathrm{Grm}$.

$\dot{\mathrm{C}} \mathrm{a}=0,021059 \mathrm{Grm} . \dot{\mathrm{C} a} \quad$. . $0,056+10$

- $\quad$ 0,182 Grm. Ammon - Magnesia - Phosphat $=$ $0,02785 \mathrm{Grm} \cdot \mathrm{Mg}=0,01707 \mathrm{Grm} \cdot \mathrm{Mg}=0,021152$

$-\quad 0,01 \ddot{A}_{1}=0,005329 \mathrm{AI} \cdot \underline{0,020692} \cdot \underline{0,07825}$

bleibt für 0,878183 Na zu berechnen . . . 1,33633

Dieser letztere Chlorgehalt entspricht $2,214513 \mathrm{NaCl}_{2}$

Das gefundene Natron - Sulfat aber • 1,745932 zusammen $\overline{3,960+ \pm 5}$ -

Die unter $\nu$ gefundene Menge an $\mathrm{Na} \mathrm{Cl}_{2}$ betrug . 3,980295

Also Ueberschuss . . . . . . . . . . 0,019850

ein Aequivalent für 0,018 einfachen, oder

0,022759 doppelt kohlensauren Natrons.

o) Da nun die Menge von $R^{\prime}$ in Ganzen . . 5,050000

betrug, die Mengen der einzelnen ausgeschiedenen

Bestandtheile sich aber folgendermassen herausstellt:

des allgemeinen Verbands der fixen Wasser-Bestandtheile noch eine grosse Aufgabe zu. lösen. Die neuere Literatur weist in dieser Beziehung mehre schätzbare, in diese Materie einschlagende, Versuche auf; auch ich gedenke darüber später einige von mir gemachte desfallsige Beiträgre zur Publicität zu bringen. Vor allem ist eine gründliche und vielumfassende positive, Entscheidung der Fragen nöthig: Welche Salze vertragen sich mit einander in sehr verdünnten wässrigen Lösungen? Bei welchem Concentrations - Grade tritt wechselseitige Zersetzung ein? Und welcher Art sind die aus diesen Zersetzungen hervorgehenden neuen Combinationen?

ㅍ. 


\begin{tabular}{lllll}
$\dot{\mathrm{K}} \dddot{\mathrm{S}}$ & $\cdot$ & $\cdot$ & $\cdot$ & 0,038880 \\
$\dot{\mathrm{Na}} \ddot{\mathrm{S}}$ & $\cdot$ & $\cdot$ & $\cdot$ & 2,123250 \\
$\mathrm{Ca} \mathrm{Cl}_{2}$ & $\cdot$ & $\cdot$ & $\cdot$ & 0,057469 \\
$\mathrm{Mg} \mathrm{Cl}_{2}$ & $\cdot$ & $\cdot$ & $\cdot$ & 0,038223 \\
$\mathrm{Al}_{2} \mathrm{Cl}_{2}$ & $\cdot$ & $\cdot$ & $\cdot$ & 0,026021 \\
$\dot{\mathrm{Na}} \ddot{\mathrm{C}}$ & $\cdot$ & $\cdot$ & $\cdot$ & 0,018000 \\
$\mathrm{Na} \mathrm{Cl}_{2}$ & $\cdot$ & $\cdot$ & $\cdot$ & 2,214515 \\
\hline
\end{tabular}

so ergiebt sich für organische Substanz und

Verlust . . . . . . . . 0,533642.

Dieses organische Princip, welches bei der Analyse fast allen Präcipitalen einem geringen Antheile nach sich anschloss, und deshaib neisten davon hinwegverglüht werden musste, ist $a \approx 0$ tfrei, und bietet alle Charactere der Humussäure dar. -

$\pi$ ) Wir wenden uns nunmehr zur Untersuchung des Rüickstandes R $(\eta)$. Er wog völlig getrocknet 3,075 Grm. Er wurde mit überschïssiger Salpetersäure angerührt, worauf man das Ganze in einem, mit einer Glasplatte bedeckten, Platintiegel (aber ohne Spuren von dadurch entbundener Hydrofluorsäure zu erhalıen) eintrocknen liess. Nach wiederholtem Anfeuchten mit Salpetersäure und späterm Zusatze von Wasser blieb reine Kieselsäure, geglüht $0,341 \mathrm{Grm}$. betragend, zurück.

९) Die salpetersaure Flüssigkeit wurde durch überflüssiges Ammon gefällt, und schnell von dem Niederschlage mittelst eines vor dem Zulritte der Luft geschützten Filters getrennt.

s) Dieser Niederschleg ward in Hydrochlorsüure gelöst, und mit reiner Aetzkali -Lösung gekocht. Das gefällte unreine Eisenoxyd löste man wieder in Hydrochlorsäure, neutralisirte die Lösung mit Ammon, fällte das Eisen als Schwefeleisen, welches seinerseits wieder zu Oxyd umgewandelt wurde. Als solches wog es.0,284 Grm. $=0,57542 \mathrm{Grm}$. doppelt kohlensauren Eisenoxyduls. 
Die rom Schwefeleisen abfiltrirte Flïssigkeit ward mit Hydrochlorsäure versetzt, vom ausgeschiedenen Schwefel (des vorhin zugesetzten Schwefelwasserstoff-Ammoniums) abfiltrirt, und mit Ammon gesältigt. Der geringe, dadurch erzeugte, Niederschlag wog $0,03 \mathrm{Gr}$. und verhielt sich vor dem Löthrohre als Kalk - (und Magnesia? -) Posphat.

v) Die alkalische, vom Eisenoxyde (s) abgesonderte, Flüssigkeit, ward mit Hydrochlorsüure gelinde übersültigt, etwas erwärmt, und mit Ammon versetzt. Eine unwägbare, von (phosphorsaurer?) Thonerde herrührende, Spur einer Trübung war die Folge dieser Reaktion.

$\varphi)$ Die ammonhaltige, eisenfreie, Flüssigkeit ron $v$ wurde jetzt durch oxalsaures Ammon gefüllt. Das gebildete Kalk-Oxalat gab geglüht 2,042 Grm. einfach kohlensaurer Kalkerde $\left(=2,9349\right.$ Grm. $\left.\dot{\mathrm{Ca}} \ddot{\mathrm{C}}_{2}\right)$.

れ) Das Filtrat ward eingetrocknet und geglüht. Der Rückstand wog 0,179 Grm. Durch Hydrochlorsäure u. s. f. liess sich daraus etwas Mangan entnehmen, das als Schwefelmangan gefällt, und durch Glühen in Oxyduloxyd umgewandelt wurde. Es wog 0,016 Grm. (=0,02/ $\mathrm{Grm}$. $\mathrm{Mn}_{2}$ ). Dadurch wird das Gewicht des obigen Rückstandes, der, nach Abzug des Mangan-Gehalts, als reine Magnesia zu betrachten ist, auf $0,163 \mathrm{Grm}$. reducirt, was $0,5{ }_{1} 5_{2} 3_{9} \mathrm{Grm} . \dot{M}_{g} \ddot{\mathrm{C}}_{2}$ gleichkömmt.

$\psi$ ) Wir hatten nun oben $(\eta)$ als $\mathbf{R}$ gefunden $3,075 \mathrm{Grm}$. und schieden aus:

$$
\begin{aligned}
& \mathrm{Si} \text {. . . } 0,341 \mathrm{Grm} \text {. } \\
& \ddot{\mathrm{E}} \mathrm{e} \text {. . . 0,284 } \\
& \text { Cि • . 0,030 } \\
& \dot{\mathrm{C}}_{\mathrm{a}} \ddot{\mathrm{C}} \text {. . } 2,042 \\
& \text {. . } 0,016 \\
& \dot{\mathrm{M} g} \ddot{\mathrm{C}} \text {. . . } 0,33_{7} \text { aus } \dot{\mathrm{M} g} 0,163
\end{aligned}
$$

Also bleibt für $\dddot{A} l$ und Verlust zu berechnen 0,025 . 
a) Zusammenstellung der erhaltenca Resultate.

Hier am Ende der chemischen Aufsuchung der fixen Bestandtheile angelangt, bleibt mir nur noch übrig, die sümmllichen qualilativen und quantitativen Ergebnisse tabellarisch zusammenzustellen. Ich werde dieser Uebersicht, damit sie möglichst grosse Bequemlichkeit zum Zwecke der therapentischen Beurtheilung unsers Mineralwassers darbieten möge, die erforderliche Ausführlichkeit widmen.

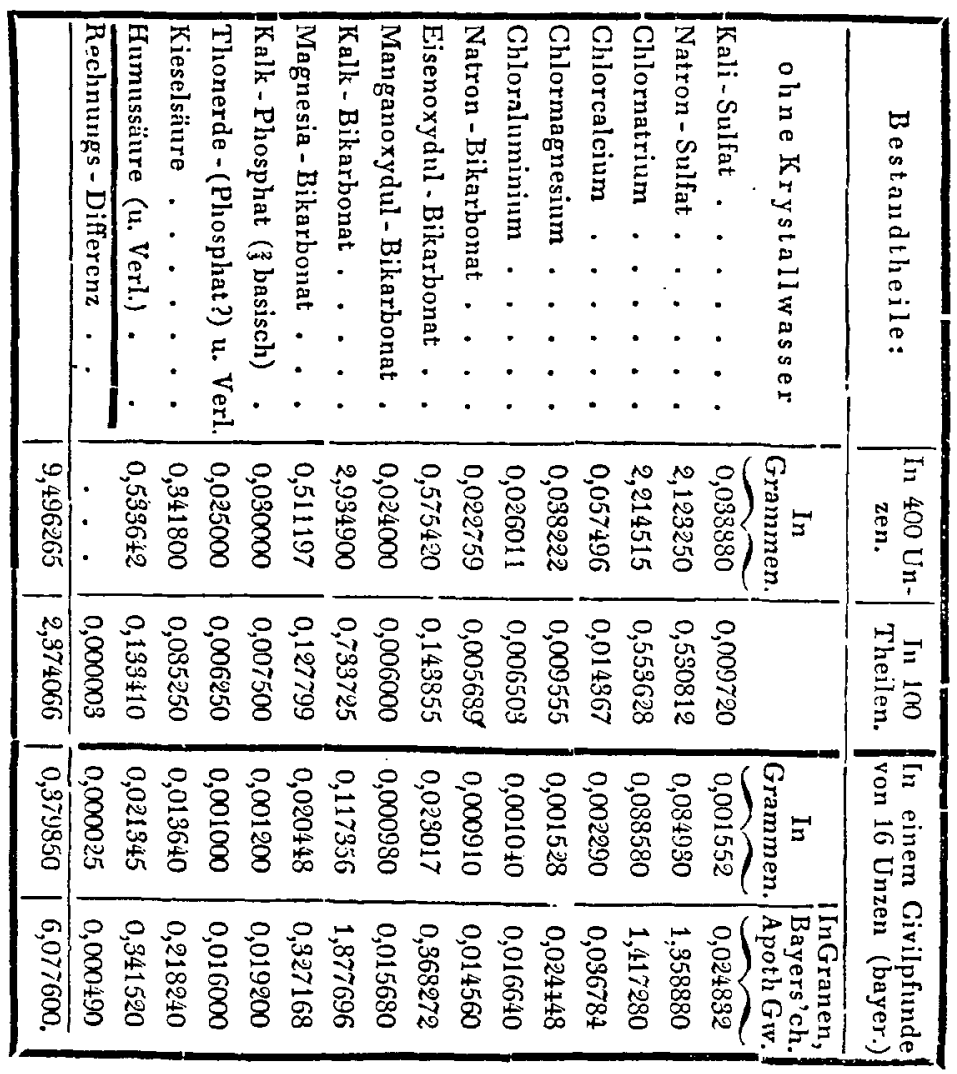


Bei der Wahrscheinlichkeit, weIche die Annahme für sich hat, dass die verschiedenen Bestandtheile der $W$ ïsser in einem allgemeinen Verbindungszustande sich darin befinden, mag auch folgende Zusammenstellung erwünscht und nützlich seyn.

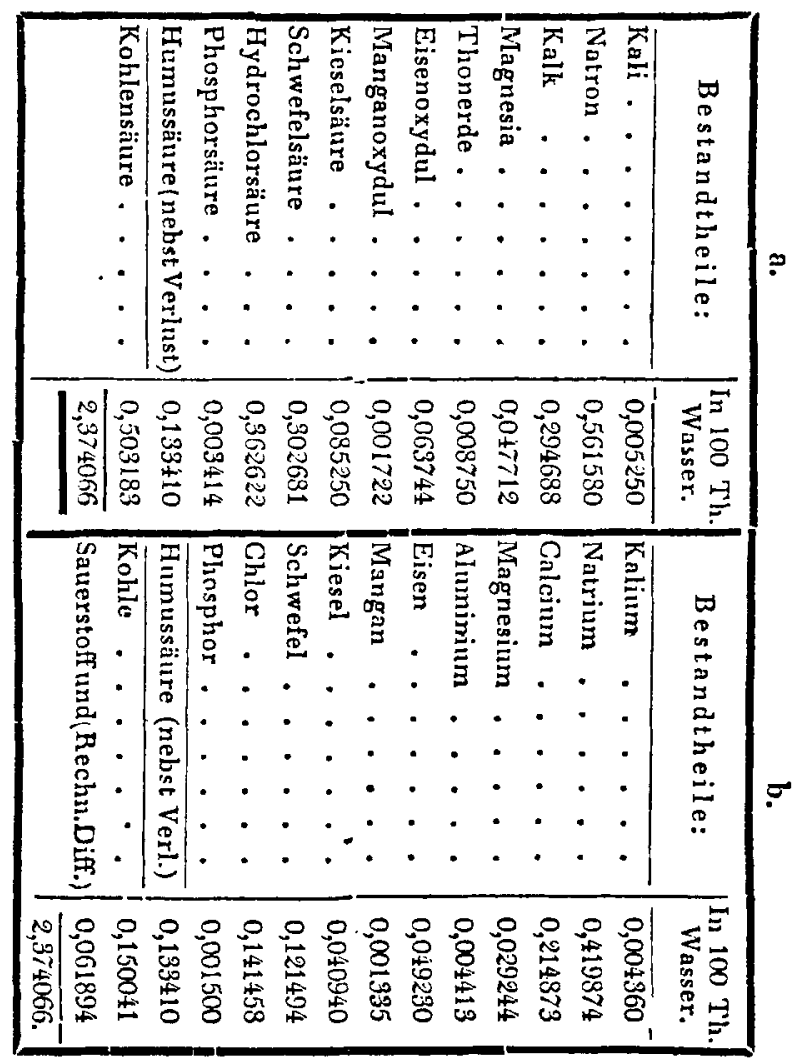




\section{$\left.\mathrm{Zu} \mathrm{B}{ }^{*}\right)$.}

Durch Erhitzen u. 8. f. liessen sich aus sechszehn Un. zen (bayer.) des mir überschickten Wassers entbinden : Baromet. - Stand Atmosph. Temp. Mitll. Temp. d. Wassers $27^{\prime \prime} 6^{\prime \prime \prime}$ rhl. $\quad+14^{\circ} \mathrm{C}$. $\quad+9^{\circ} \mathrm{C}$.

Gesammter Gasgeh. Kohlensäure. Atmosph. Luft.

In rheinl. Kubik - Zollen.

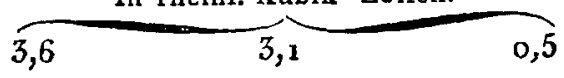

Die zu drei wiederholten Malen angestellten Versuche blieben in ihreu Resultaten constant. Hydrolliongasgehalt ist, wie ich bereits erwähnte, nur im Wasser einiger, nicht sämmllicher, Flaschen spurweise bemerkbar gewesen, und konnte deshalb nicht bestimmt' werden.

Welcher Grad von Werth äbrigens diesen pneumatischen Resultaten zuzuerkennen seyn dürfle, glaube ich oben wahrbeitsgemäss und genïgend auseinander gesetzt zu haben. -

\section{Schlussomerkungen.}

Das untersuchte Wasser vereinigt, nach den vorausgeschickten chemischen Ergebnissen, in sich die Eigenschaften eines (kalten) Eisensäzuerlingrs, dessen Zusammensetzung eine nicht ungünstige Prognose rücksichtlich seines medicinischen Werlbs zu stellen gestattet.

Erwägt man hiebei, dass sich der Gehalt des Wassers an ruïtzlichen Bestandtheilen bei vervollkommneter Quellen Fassung noch steigern dürfte; erwägt man ferner die unge-

*) Die pneumatischen Versuche wurden gerade so, wie bei meiner Analyse der Ueberlinger Heilquelle, ausgeführt. Vergl. meine Schrift: „Ueberlingen und seine Heilquelle; Constanz, W. Wallis, 1831. 8"“6; und ,Kastner's Archiv fürr Chemie und Meteorologie II, $279 \mathrm{ff."}$

H. 
wölnnlichen Vorzüge der Lage und Umgebungen Dürkheims, die Annehmlichkeit der Nachbarschaft grösserer Städte, wie Mainz und Mannleim, so wie des Regierungssitzes Speyer; und reilen sich endlich in der Folge diesen Vortheilen noch ausgedehntere Bad-Einrichtungen mit gleichzeitiger Benulzung der Brücken - und Soolquellen an: so darf man kaum daran zweifeln, in Dürkheim noch eine Mannigfalligkeit von Gästen begrüssen zu können, die der Badekur, oder der jetzt so beliebt gewordenen Traubenkur, ihre Huldigung darbringen.

\section{Ucber die Bereitung des Eisenprotojodids und Kaliumjodids ; \\ rom \\ Dr. du Mênil.}

Das Eisenprotojodid, welches in neuerer Zeit dem Arzneischatz einverleibt ist, zeigt sich als ein Präparat, welches viel Sorgfalt bei seiner Bereitung erfordert, indem es sehr leicht in Sesquijodid übergeht. Hat man nämlich vorschriftsmüssig Jod mit der Hälfte seines Gewichts an Eisenpulver zusamnuengerieben und dann mit $3 \mathrm{Th}$. Wasser zu einem Brei gemacht, die Erhitzung abgewartet, das Ganze eine Zeillang warm gehalten, die Flüssigkeit noch etwas verdünnt und auf ein Filter gebracht, so muss das grüne liltrat, wenn es ein reines Salz geben soll, sogleich in einem Destillirgefüsse abgeraucht und von der atmosphärischen Luft ausgeschlossen zur Kryslallisation gebracht werden, geschieht dieses an offener Luft, so findet man die Krystalle mit Eisenoxyd überzogen und die Mutlerlauge enthält grüsstenlheils Eisensesquijodid. Da nun letzlere Uebelstände bei Bereitung kleiner Mengen dieses Präparats nicht wobl zu vermeiden 